\title{
Usability testing with children: an application of Pedactice and Ticese methods
}

\author{
Alessandra Carusi ${ }^{\mathrm{a}}$, Cláudia Mont'Alvão ${ }^{\mathrm{a}}$ \\ ${ }^{a}$ Ergonomics Laboratory, Arts \&Design Department, Pontifical Catholic University of Rio de Janeiro, PUC-Rio, \\ Rua Marquês de São Vicente, 225, Gávea - Rio de Janeiro, RJ - Brazil
}

\begin{abstract}
Graphic representation of clickable areas in computer interfaces should allow mental representations that facilitate system's navigation during the cognitive process. If it doesn't happen users can feel lost. When thinking about websites designed to children with both entertainment and educational intention these systems must stimulates this kind of users. As many other activities for children nowadays, many system are commonly used as support for contents explored in school, as a complement. The design of theses educational systems must have the compromise to allow these users navigate and feel capable to explore every part of these interfaces. This paper is focusing the results of Ticese and Pedactice methods and shows the importance of these results as an exploratory phase in a bigger research that evaluates interfaces with children. Quantitative and qualitative data were collected related to structuring the user's mental model, influenced by the graphic representation of hypertext areas in a children's educational site.
\end{abstract}

Keywords: educational site, navigation, design, graphic representation

\section{Introduction}

How to adequate navigation systems, when considering those designed for children? This question was the starting point for this research considering the design of clickable areas, which mean navigation and interaction to users in hypermedia systems.

One action includes the interconnections among cognition and human behavior [1]. User's actions regard to motivation, external behaviors and mental processes that are combined and organized to reach an objective. According to the authors, this question highlights the necessity of considering the observed behavior while studying human-computer interaction: they emphasize that human behavior must be studied not only considering stimulusresponse approach, but also a cognitive relation to the external behavior.

When thrashing out the relation between cognition and external behavior, it is possible to realize interface design as a main responsible for system navigation compatibility to users' task. Graphic elements influence the cognitive process, helping to
" punctuate informative characteristics that are relevant and allow that the architecture of this information can be accessible to the users [5]. Clickable areas usually represent linked ideas creating a network of meanings and showing to user the options while navigating in the system. Therefore, system' model, developed by designers could be similar to users' mental model, what improve their interaction with the system.

Graphic representation of clickable areas in computer interfaces should allow mental representations that facilitate system's navigation during the cognitive process. If it doesn't happen users can feel lost.

When thinking about websites designed to children with both entertainment and educational intention these systems must stimulates this kind of users. As many other activities for children nowadays, many system are commonly used as support for contents explored in school, as a complement. The design of theses educational systems must have the compromise to allow these users navigate and feel

\footnotetext{
Corresponding authors: alessa.carusi@gmail.com; cmontalvao@puc-rio.br
} 
capable to explore every part of these interfaces. Hypermedia environments are usually known in Learning environment. However flexibility and nonlinearity of these systems has been seen as main causes for confusion and disorientation by users. Due these characteristics users sometimes don't know where they are or where to go inside the system. To handle these inherent conditions hypermedia users adopt specific strategies. They must know where they are and decide which will be the next step to create a cognitive representation in this net content [5].

So, in the context of children's' websites it is easy to understand the importance of understand the mental model of these users, considering their cognitive processes relating to clickable areas and behavior when navigation in computer systems.

\section{Methodological approach}

To aim the research objectives, both qualitative and quantitative data were collected referring to users mental model, influenced by graphic representation of pre-selected clickable areas in a determined children's website. At a later time, aspects of users models where compared to usability models in the chosen system.

The methods defined for the search data collection include different subjects - both the experts and children evaluation.

The experts' evaluation was divided into two parts:

- $\quad$ the first was targeted to educators, through a questionnaire about the site, based on Pedactice method;

- and the second, targeted to interactive systems designers, through a checklist for evaluating the rate of ergonomic line site, based on Ticese method.

In the evaluation with children, we used the codiscovery method that allows children participation as their understanding and their behavior in using the system during the study. This paper is focusing the results of Ticese and Pedactice methods and shows the importance of these results as an exploratory phase in a bigger research that evaluates these interfaces with the users. Quantitative and qualitative data were collected related to structuring the user's mental model, influenced by the graphic representation of hypertext areas in a children's educational site.
Facing those criteria was chosen Kiagito website, which is part of the $E d u K b r$ portal with interactive activities for first phase of elementary school classes. Focusing in children, the main objective of the website is to stimulate learning, not only as an intellectual activity, but also pleasurable, where the leisure acts as a motivational aid in this process.

\section{Ticese method and application}

We used Ticese (Inspection Technique of Ergonomic Conformity in a Learning Software Técnica de Inspeção de Conformidade Ergonômica de Software Educacional) method in order to establish integration between the ergonomic criteria of usability inspection with criteria for evaluating educational websites and software. The technique consists of a checklist applying, whose main goal is to provide evaluators involved in the selection process of products with education quality [4].

The Ticese composition was the collection of information through a literature consultation, involving heuristics and ergonomic teaching, and also in opinions collection from experts in usability, pedagogy and psychology. The method was validated by people with knowledge and software evaluation practical, which implemented and expressed their opinions. With the results of this process, a checklist was created and adapted to provide facilities in its application. When applied, this tool indicates the rate of ergonomic conformity, which indicates the pedagogical aspects and the aspects related to the interactive educational system interface that need to be more appropriate to the users group.

The ergonomic conformity analysis in the educational software evaluation is justified because the educational software aims to assist in the teaching/learning process of a particular content. For this, the following features must be present in this system:

- It is easy to use;

- It is easy to understand;

- promoting the assimilation of content;

- have motivational aspects that keep the user's attention;

- be able to attract and capture the users' interest;

- verify the students understanding degree, as well as their difficulties.

All these resources must be provided in an interface that allows users to easily interact with the 
system, so they can focus their attention only on the educational objectives to be achieved.

To the Ticese application, the evaluator should have the method and the system's recognition. Thus, the participant must have a first contact with the system in order to understand its operation, in addition to a prior reading of the criteria set definitions, the Form of Inspection and quantitative treatment. For this research 9 criteria were investigated, corresponding to closed questions [4]:

- Conduction: refers to means to advise, orientate, inform and guide the user while interacting with the computer (messages, warnings, labels etc);

- Adaptability: regards to system capability to react according to the context and also user's necessities and preferences. An educational system can't fit its target due individual differences. As many ways a certain task could be performed, larger will be the option for a user to choose and dominate the tasks while learning. In this way, different procedures and options must be offered among these different users, that can choose how to reach a same objective;

- Explicit control: refers to system explicit processing related to users actions, and also to users 'control about the system. In this way, errors and ambiguities are limited and system could be more acceptable for users;

- Resources to support the comprehension of contents: this criteria refers to the support that system offers to user to understand the pedagogical contents. Multimedia, motivational and learning checking resources, contributes to this aim. System must promote stimulating situations to the students, not only catching their attention but also mainlining attention during the whole interaction;

- Errors management: these are the mechanisms that avoid or reduce errors occurrence. Ticese technique considers as system errors: wrong data insertion, inadequate data format, and inadequate answers as knowledge acquisition and verification;

- Evaluation of learning: this criteria deal with the learning verification of the presented contents. It is important that system presents pedagogical concepts that are learned by students;

- Workload: regards to all interface elements that plays an important role in reduce the cognitive workload and increase the dialogue efficiency. As much is the workload, bigger will be the probability to make mistakes and longer will be the learning.;

- Meanings: refer to adequacy among object, presented or asked information and its reference, it means, the interpreted meaning for the user. Meaningful terms to the user could result in conduction problems that can lead to incorrect option selection. When coding is adequate to user repertory, recall and recognizing are more frequent.

We collected data from questionnaires answered by 11 designers and experts in human-computer interaction. Participants have around 12 years of experience, and all of them have already designed educational websites. In this group, four have Master degree in Human-computer interaction area.

\subsection{Results}

The problems report indicated by the evaluators emphasizes the care with the interfaces development focused on educational environments.

Ticese results shown that in Kiagito website' interfaces communication problems were previewed considering navigation areas and users. These problems can be caused by graphic and conceptual representations options that are offered by the menu and images in the website.

And also, taking into account cognitive differences among child and adult users these questions can indicate difficulties that could be bigger than the one first imagined, once these evaluations were carried out by adults.

The results of the answers and final comments of the evaluators pointed out problems related to their criteria. One of the website areas that were often cited are the navigation menu; graphic elements in internal pages presenting consistency problems and a excessive number of pop-up windows.

Even if Ticese hadn't the specific approach in any website area, its results emphasize that the areas considered by the navigation criteria must be reviewed.

\section{Pedactice method and application}

The method Pedactice is defined by the application of a questionnaire with open answers, with a pedagogical approach, it means, directed at teachers/educators. This focus on teachers is justified by the role that these professionals play as support and orientation to these students/users when using the technology inside or outside schools [2]. Even Pedactice has it focus on teachers, it is not limited to pedagogical aspects [3]. This questionnaire also involves usability criteria using accessible terms, usual in their context.

Once the educational software is many times 
developed by a designer, and not an educator professional, a critical analysis must be done about the relation between designers and educators, and also the expectancies of integration and relationship of its utilization [3]. This argument can be extended also to educational websites.

An evaluation of an educational product should consider not only the product itself. It must be extended to real use situation analysis, it means, the context of learning and knowledge acquisition. In the scope of educational products centered evaluation results depend on the nature of learning and tasks requirements (didactical objectives), students cognitive skills and knowledge and contextual factors. The evaluation of these products must consider all theses aspects [2].

That application of this questionnaire considers three main aspects [3]:

- the product, emphasizing systems characteristics (interface, navigation, usability);

- its utilization, focusing on context of use and main users (students and teachers);

- results of learning, observing content.

The subjects/participants that answered Pedactice questions were twelve teachers. An initial meeting with the teachers occurred, when the research was presented, and they were concerned about its objectives, also the procedures of this evaluation technique, named Pedagogical Potencial Synthesis Formulary (Ficha Síntese de Potencial Pedagógico). This meeting also served to explain what should be observed by them, i.e., get acquainted with the studied website. At last, was asked during the observation that teachers should do their personal notes about relevant facts to a posterior questionnaire fulfillment. Hereupon, each teacher, based on the criteria shown at the Pedagogical Potencial Synthesis Formulary observed his/her own group of students in the context of usage the studied website Kiagito. At this moment, children behavior, their facility and difficulties of usage while using the website.

After their experiences with the Kiagito website, each teacher answered about their observation when students were navigating in this website, some considerations about it overall quality, and the pedagogical potential of this website.

\subsection{Results}

After data collection and analysis of the twelve questionnaires answered by the participant teachers was possible to analyze the quantitative data of
Pedactice technique, and also the comments related to the opened questions.

Considering the overall Pedactice results, it was concluded that teachers considered Kiagito website as a hypermedia system, which aims literate children from 7 to 10 y.o. However, results shown that teachers evaluate that the website design don't deal in a proper way with this age range - there isn't an ideal difference among age and development for proposed tasks.

Website was also considered broad of knowledge contents areas. Teachers indicate 14 disciplines that could be approached by this website, highlighting its multidisciplinary potential.

Teachers also indicated and exemplified system and content failures that could affect the credibility of the overall website.

The website was considered useful and relevant when taking into account the didactical activities.

When considering interfaces, teachers evaluate them as aesthetically pleasant, but partially intuitive, consistent and motivating.

Finally, areas that were emphasized as more difficult for navigation comprehension were the sections Clubinho and Salão de Jogos. These areas present activities that were considered complex to the children, but at same time, once they are related to online games, were also the most searched inside the system.

\section{Conclusion}

The initial question of this research was the adequacy of navigation systems in educational websites for children as users focusing the design of clickable areas, which highlight the navigation and interaction options in hypermedia systems. .Users interaction is enhanced when system model is similar to users mental model.

An educational website has the following specificity: the success of learning process results from the utilization of a aware usage by a motivated user.

Reach objectives of learning depends on the incentive that system gives to user to think and has critical sense of their action while using the system. To that, children must feel stimulated and confident to follow a path inside the website until he/she finds the desired information. It means that children must know what he/she is doing, feeling pleasant and curious to search for information. 
Clickable areas can stimulate users in learning process, retrieving information and resolve problems that can leads to satisfaction and motivation. These graphic representations are subject to a cognitive process, which meaning will impact directly in user behavior. The efficacy of clickable area in interactive system navigation depends on its graphic representation, i.e., its design.

Exploratory phase clarified the relevance and relevance of detected problems.

Results of both methods Pedactice and Ticese were similar in some aspects:

- website interfaces were not considered motivated enough to stimulate students for interaction with the presented pedagogical content;

- participants didn't consider an "easy-to-use" interface, that were considered partially intuitive and consistent.

- main menu was considered low expressive, presenting tags that sometimes were not appropriate to their contents;

- an excessive number of pop-ups disturb navigation.

Then, one can conclude that in the experts' eyes, the navigation design cannot be based on users' infant cognitive characteristics. Although, the site presents a personages and histories scene knows by the users, the motivation for tasks accomplishment can be harmed in way that the child does not understand its navigation of the site. Therefore, it is evidenced greater care in the design process of educative websites, as form to involve the navigation and the systems tasks resolution.

Pedactice technique results allowed the researcher the understanding of teachers' point of view about the Kiagito website, once teachers observed their own students, and not the researcher. This view was essential to obtain pedagogical information about the context and to confirm which age range was considered appropriate to a future participatory evaluation.

Teachers considered the website as an important didactic tool, even they emphasized some system failures and the necessity of this website be improved, as a differential from other similar websites. They also noticed lack of consistency in some interfaces.

Besides that, they observed that most clicked areas were those related to games and activities for "creation", as Clubinho and Salão de Jogos. These areas were also considered the most complexes once they present some unknown words and graphic representations (list box, for example).
Considering Ticese technique application, was possible to collect important data about design and human-computer interaction about Kiagito website. This technique was essential to understand designers point of view that develop educational interfaces. In a general evaluation, this website wasn't considered as easy to learn and the main aspects related were: navigation menu considered as "not representative" and labels that don't match its contents, and an excessive number of pop-up windows.

Collected data and the analysis of the exploratory phase were fundamental to organize a participatory phase including children. Graphic elements that showed clickable areas were the scope of this research, but it was also necessary to determine other relevant aspects to users, the most complex ones. The contribution obtained by volunteers in Pedactice and Ticese techniques was relevant to choose the website parts to be studied with children in the next phase.

\section{References}

[1] Bedny, G.; W. Karwowski; and M Bedny, The Principle of Unity of Cognition and Behavior: Implications of Activity Theory for the Study of Human Work, in: International Journal of Cognitive Ergonomics 5 (4), 2001, pp 401-420

[2] Costa, F., Contributos para um modelo de avaliação de produtos multimédia centrado na participação dos professors, in: Anais do 1o Simpósio Ibérico de Informática Educativa. Aveiro, PT, 1999

[3] Freire, L. L., Navegação e design em softwares educativos: uma abordagem ergonômica, Ms.C. Dissertation. Recife: Universidade Federal de Pernambuco, 2005

[4] Gamez, L.: Ergonomia escolar e as novas tecnologias no ensino: enfoque na avaliação de software educacional. Dissertação - Ms.C. Dissertation. Braga, PT: Universidade do Minho (1998)

[5] Puntambeakar, S.; Stylianou, A.; Hübscher, R., Improving navigation and learning in hypertext environments with navigable concept maps, in: HumanComputer Interaction 18, 2003, pp 395-4 